\title{
PRINCIPALES CAUSAS DE PORQUE LA POBLACIÓN MEXICANA PROCRASTINA EN SU SALUD
}

\author{
MAIN CAUSES OF BECAUSE \\ THE MEXICAN POPULATION \\ PROCRASTINA IN \\ YOUR HEALTH \\ Recibido 17 de noviembre \\ Aceptado 19 de diciembre 2015
}

Correspondencia: M. en C. Adela Alba Leonel Antiguo camino a Xochimilco y Viaducto Tlalpan Col. San Lorenzo Huipulco C. P. 14370

Tlalpan, México, D. F. Tel. 55562332 Ext. 269 adelaalbaleonel@yahoo.com.mx

\section{Autores:}

Linda Gloria Granados Villanueva

Pasante de Servicio Social de la Licenciatura en Enfermería y Obstetricia. Escuela Nacional de Enfermería y Obstetricia, UNAM

M. en C. Adela Alba Leonel

Mtra. en Ciencias Sociomédicas con énfasis en Epidemiología. Profesor de Carrera Asociado "C", Escuela Nacional de Enfermería y Obstetricia, UNAM

Palabras clave: Procrastinación en salud, factor de riesgo, calidad de atención

Key words: procrastination in health, risk factors, quality of care 


\section{RESUMEN}

Introducción: La procrastinación en salud es un factor de riesgo para que se presenten tanto enfermedades agudas o crónico degenerativas, así como complicaciones en general.

Objetivo: Identificar las principales causas de procrastinación en salud de la población mexicana.

Metodología: El presente estudio fue de tipo cuantitativo descriptivo, la unidad de observación fue pacientes y personas que acudieron a un hospital de tercer nivel de la Ciudad de México y en población abierta, el muestreo fue no probabilístico. Se aplicó un cuestionario exprofeso "Escala de procrastinación en la atención de la salud" con 14 reactivos.

Resultados: La muestra total fue 317 personas (200 en un hospital y 117 en población abierta). El 58\% correspondió al sexo femenino, el 56\% tenían entre 18 y 39 años de edad. El 39\% cuentan con seguro popular, el 16\% no tienen ninguno. El 99.3\% procrastina en su salud. Las principales causas de procrastinación fueron: el 64\% posterga ir al médico porque los servicios de atención son muy lentos, 14\% refiere que no le resuelven su enfermedad, de estos el $42 \%$ lo complementa con remedios caseros; el 17\% no termina el tratamiento por cuestiones económicas; el 38\% asiste cuando ya tiene una complicación. El 50\% se atiende en último momento por no tener tiempo para su atención; 27\% por perder mucho tiempo para ser atendido, 25\% por no tener dinero y el 20\% le da miedo saber qué es lo que tiene.

Conclusiones: La procrastinación en salud se debe en gran medida a la falta de calidad en la atención de los servicios de salud como tiempo de espera, resolución de la enfermedad y accesibilidad al tratamiento.

Palabras clave: Procrastinación en salud, factor de riesgo, calidad de atención.

\section{ABSTRACT}

Introduction. Introduction: The procrastination in health is a risk factor for both acute and chronio degenerative diseases, as well as complications arise in general.

Objective: To identify the main causes of procrastination in health of the Mexican population.

Methodology: This study was quantitative descriptive, the unit of observation was patients and people who came even from Mexico City and in open population tertiary hospital, sampling was not probabilistic. A questionnaire was applied indifferent reagents 14 "scale of procrastination in health care".

Results: The total sample was 317 people (200 in a hospital and open population 177). 58\% corresponded to females, 56\% were between 18 and 39 years old. The 39\% have popular insurance, $16 \%$ have none. The $99.3 \%$ procrastina in your health. Were the main causes of procrastination: the $64 \%$ delayed going to the doctor because services are very slow, $14 \%$ concerned that not they solve their disease, these $42 \%$ complements it with home remedies; 17\% does not end treatment for economic reasons; the 38\% assists when you already have a complication. $50 \%$ served at last moment by not having time for your attention; $27 \%$ by lose much time to be served, 25\% for not having money and $20 \%$ gives you fear know what they have.

Conclusions: Procrastination in health is largely due to the lack of quality of care in health services as time waiting, resolution of disease and treatment accessibility.

Key words: procrastination in health, risk factors, quality of care.

\section{0}

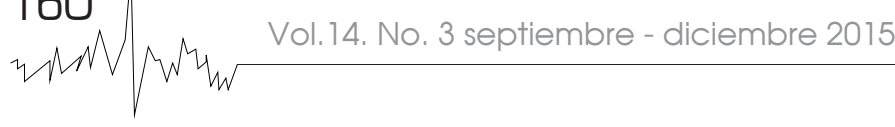




\section{INTRODUCCIÓN}

a palabra procrastinación que usualmente escuchamos, proviene de "procrastinate", que significa "pro" hacia adelante, "cras" o "crastinus" para mañana, lo que significa aplazar intencionalmente o habitualmente algo que debería ser hecho.' Del latín: pro, adelante, y crastinus, referente al futuro) o posposición, es la acción o habito de postergar actividades o situaciones que deben atenderse, sustituyéndolas por otras situaciones más irrelevantes y agradables. ${ }^{2}$

Este es un fenómeno habitual de las actividades humanas, que data desde hace varios siglos, tanto en el oriente como en occidente. ${ }^{3}$ Actualmente esta acción es típica en las sociedades industrializadas; tanto, que se le ha considerado un "mal moderno".4 Generalmente la postergación es producida por la falta de hábitos para abordar situaciones en las que debe focalizar la atención - bien darle prioridad a lo inaplazable. ${ }^{5}$ Habitualmente las personas que posponen decisiones de cualquier índole desarrollan alteraciones emocionales como: ansiedad, frustración, apatía, resentimiento, culpa, vergüenza, autodesprecio, falta de autoestima y depresión, como resultado de la falta de esfuerzo para afrontar las consecuencias negativas de evitar o demorar las situaciones o tareas. 6.7

La procrastinzación se encuentra en diferentes ámbitos, sin embargo el que más se ha estudiado es en el nivel académico. Aun cuando existen otros muy distintos como el laboral, cuidado de la salud, atención de la familia o pareja, en el desarrollo y capacitación profesional e incluso en el uso del tiempo libre. Existen evidencias aisladas de investigaciones como en meta análisis y revisiones sistemáticas efectuadas sobre miles de casos particulares que sugieren que el 89\% de las personas manifiestan procrastinación en distintos niveles y áreas.

En el presente artículo, nos enfocaremos a la procrastinación en salud aun cuando existe poca la bibliografía que lo argumente o haga notar en cuestiones de salud. Nos podemos percatar que día a día asciende el número de personas que postergan su estado de salud, o bien actividades que ayuden a prevenir o evitar enfermedades como: la realización de chequeos médicos, exámenes de laboratorios, modificar su estilo de vida, adherencia al tratamiento, etc.

El procrastinador en salud sabe que debe cuidarse, que debe hacerse chequeos médicos, sabe que puede y trata de hacerlo, pero en realidad no lo hace ya que en su camino encuentra obstáculos y barreras que le impiden culminar con esta tarea, por lo que es importante identificar cuáles son las principales causas de procrastinación en su salud.

Quizás una de las razones por las que no se atienden es porque aún no se han manifestado algunos de los signos y síntomas de la enfermedad debido a que se encuentra dentro del estadio inicial, lo que lleva a aplazar la atención hasta el grado en que ya no hay más que hacer debido a que se encuentra en una etapa terminal que es cuando ge neralmente se presentan malestares mayores o bien los signos y síntomas son más visibles, o cuando se trataron con "remedios caseros" y estos no tuvieron el efecto esperado.

Generalmente la población no considera o cree conveniente realizarse examen médico general con el propósito de realizar detección oportuna, prevenir enfermedades así como tratamiento oportuno.

En ocasiones el exceso de confianza y/o el aparente control sobre la salud o enfermedad, son algunas de las causas de procrastinación en salud, así como la falta de tiempo, la apatía, siendo estos un factor de riesgo para presentarse la enfermedad, complicaciones y en ocasiones la muerte.

Por otro lado la procrastinación puede ser el reflejo de características ambientales como es la atención en los servicios de salud con respecto al tiempo de espera, falta de recursos, etc. que lo motiva a postergar la atención a su salud; además de características personales que promueven el aplazamiento,

Vol. 14. No. 3 septiembre - diciembre 2015 Wh 
vinculadas con una baja motivación al logro.? Steel explica las causantes de procrastinación por falta de motivación la cual es afectada por cuatro factores:

- Expectativa: Esperan terminar la tarea ya sea fácil o difícil, existen personas con exceso de confianza en sus capacidades para sacar adelante ciertas tareas. Lo cual provoca que pospongan sus obligaciones hasta el último momento y en ocasiones obtiene un gran éxito. Sin embargo en el caso de salud consideran que la consulta médica les resulta muy difícil por diferentes situaciones, por lo que tienden a procrastinar.

- Valoración: No todas las tareas que realizan tienen el mismo valor y no resultan igualmente placenteras. La mayoría de las personas muestran una mayor tendencia a procrastinar cuando los plazos de expiración se encuentran más lejanos en el tiempo. Si el tiempo de espera para la atención en salud fuera corto las personas acudirían con mayor frecuencia teniendo así una mejor prevención y control de enfermedades y/o complicaciones, generando en la población mayor interés en su salud.

- Impulsividad: Hacer la actividad sin reflexionar, simplemente lo hace por la emoción del momento. En caso de salud podría ser que cuando hay campañas de detección como examen de Papanicolaou, las personas se hacen el estudio sin embargo, no van por los resultados porque estos los entregan tiempo después.

- Demora de la satisfacción: No es lo mismo saber que la tarea terminará en 15 minutos, o que disfrutaremos de los beneficios inmediatamente. Con respecto a la salud cuando ya existen signos y síntomas los pacientes piensan que tan solo ir a la consulta se curaran, sin embargo es necesario seguir el tratamiento por unos días, a veces los pacientes creen que tan solo tomar una sola vez el tratamiento se van a curar y no consideran que esto depende de la patología si es curable o no así como su estadio ${ }^{2}$
Si relacionamos lo anterior con las carencias de los sistemas de salud en México, con respecto: tiempo de espera para ser atendido, falta de recursos materiales y humanos, resolución del problema de salud, carencia de medicamentos, costos de las consultas y calidad de atención. Aunado a las características propias de cada individuo, podrían ser algunos de las causas de porque la población tiende a postergar su salud.

La procrastinación en salud en cualquiera de sus formas es un problema de salud pública, el cual tiene un impacto económico en el paciente, población y en los sistemas de salud, ya que aumenta la prevalencia e incidencia de las enfermedades agudas/crónicas, complicaciones de estas e inclusive la muerte, aumentando así los días de estancia hospitalaria, recursos materiales y humanos en salud.

De acuerdo con la Encuesta Nacional de Satisfacción de los Servicios de Salud del año 2000 se reportó que uno de cada cuatro mexicanos había postergado su atención médica, por problemas económicos. Por lo que a través del Programa de Salud para Todos se promueve el Seguro Popular de Salud (SPS) con el propósito de brindar protección financiera a la población que careciera de seguridad social en salud y reducir los gastos de bolsillo en que incurren las familias por atender su salud y que constituyen una causa de empobrecimiento para las familias más vulnerables, así como fomentar la atención preventiva de la salud. ${ }^{8}$

\section{METODOLOGÍA}

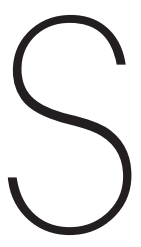
e realizó un estudio cuantitativo descriptivo de tipo transversal, la unidad de observación fue pacientes y personas que acudieron a un hospital de tercer nivel del D.F. así como en población abierta, el muestreo fue no probabilistico.

Los criterios de inclusión fueron personas y pacientes de ambos sexos mayores de 18 años de edad que acudieron aún hospital de tercer nivel de la

\section{2} MNMVWhy vol.14. No. 3 septiembre - diciembre 2015 
Ciudad de México así como población aledaña a este; criterios de exclusión: personas menores de edad y criterios de eliminación: que no hayan respondido el cuestionario correctamente y no quisieran participar.

Se aplicó un cuestionario exprofeso "Escala de procrastinación en la atención de la salud" con 14 reactivos diseñado por Granados Villanueva Linda Gloria y Alba Leonel Adela, el cual contemplaba datos demográficos (sexo, edad, escolaridad, ocupación y derechohabiencia) y 14 reactivos específicos de causas de procrastinación en salud,

\section{RESULTADOS}

a población fue de 317 personas (200 en un hospital y 117 en población abierta). En cuanto a los datos sociodemográficos, el 58\% correspondió al sexo femenino, el 56\% tenían entre 18 y 39 años; el 53\% trabaja actualmente; 39\% de la población cuenta con seguro popular y el 16\% no cuenta con servicios de salud (ver cuadro I).

El 99.3\% de la población reporto que procrastina en salud. El 18\% afirmo que siempre cumple ir al médico cuando decide atenderse, solo el 55\% de la población refiere realizarse chequeos médicos cada 6 meses, y el 62\% reporto ir al médico antes de una complicación.

En lo que respecta a las características de porque la población mexicana procrastina en su salud, esta se analizó por dos grandes rubros: 1) los servicios de salud y 2) por las características propias de la población. el instrumento fue aplicado de forma individual con un tiempo de respuesta entre 10 y 15 minutos, los datos fueron recolectados en el mes de julio de 2015.

En lo que respecta a los aspectos éticos, se les explico a los participantes que su participación era voluntaria, anónima y que la información se manejaría únicamente de forma general donde se respetaría su privacidad y confidencialidad y solo se utilizaría con fines estadísticos.

La información se capturo y analizo en Excel, se realizó un análisis descriptivo y relativo para cada variable de la investigación.

Cuadro 1. Datos sociodemográficos de la población entrevistada, 2015

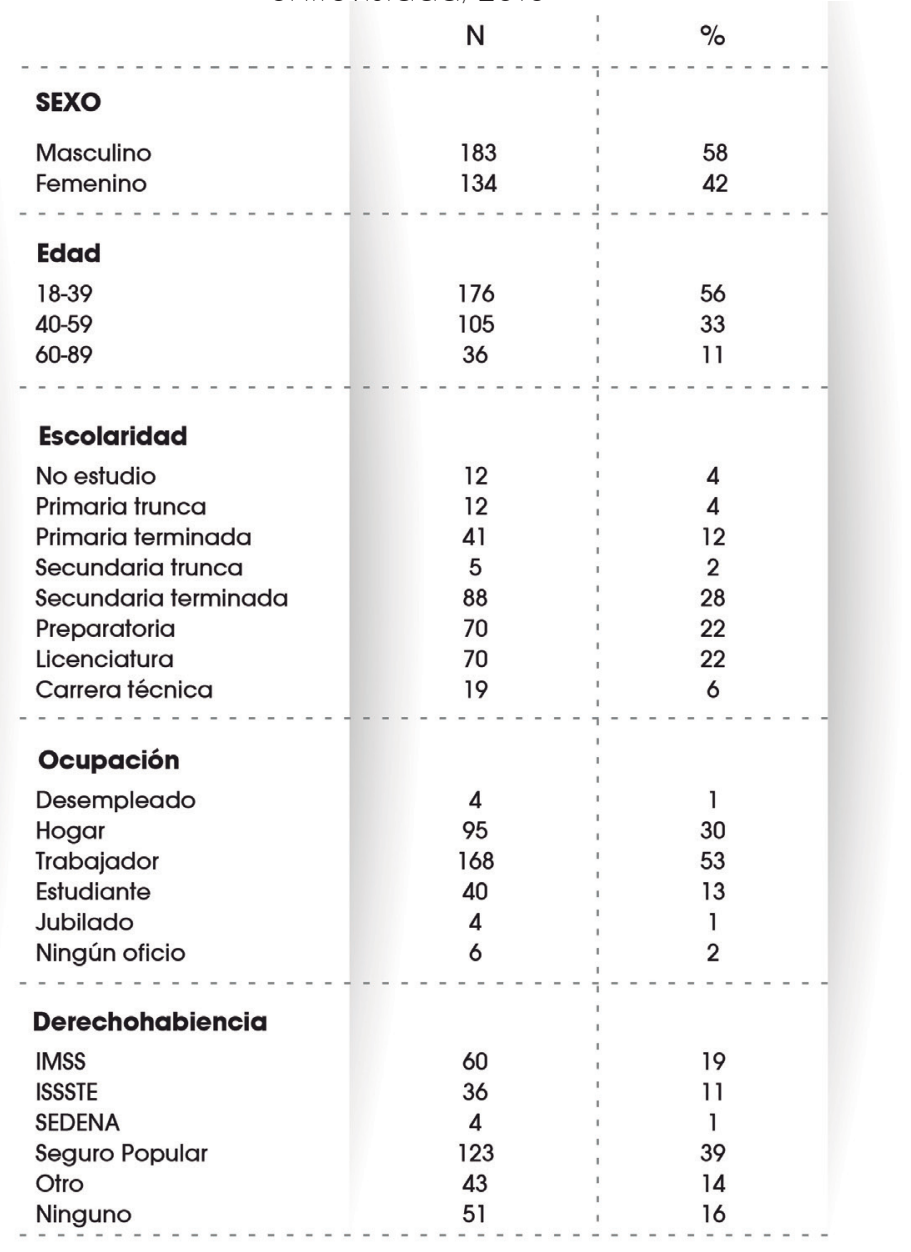


En lo que respecta a los servicios de salud, la población reporto que el 64\% posterga ir al médico porque son muy lentos, 27\% pierde mucho tiempo para ser atendido y el 14\% reporto que no le resuelven su problema de salud. Así como el alto costo de la consulta y del tratamiento (ver cuadro II).

Quizás una de los principales características de la población a la cual se le pregunto porque procrastina en su salud es que el 50\% se atiende en último momento por no tener tiempo para su atención, 26\% porque no tiene dinero para atenderse, 20\% le da miedo saber qué es lo que tiene; el 50\% reporta ya sentirse mejor, el 56\% de la población les disgusta tener que modificar su dieta y estilo de vida (ver cuadro III)

Cuadro 2. Porque la población de un hospital de tercer nivel y población abierta del D. F. procrastina en su salud, 2015.

\begin{tabular}{lcll}
\multicolumn{1}{c}{ Población } & $\%$ & \multicolumn{1}{c}{ Servicios de salud } & $\%$ \\
\hline No tiene tiempo & 50 & \multicolumn{1}{c}{ Son muy lentos } & 64 \\
\hline No tiene dinero & 26 & Pierde mucho tiempo para ser atendido & 27 \\
\hline Miedo a saber qué es lo que tiene & 20 & No resuelven el problema de salud & 14 \\
\hline Se siente mejor & 50 & Son muy caras las consultas & 11 \\
\hline Le disgusta modificar la dieta & 56 & El tratamiento es caro. & 9
\end{tabular}

Cuadro 3. Causas por las que la población de un hospital de tercer nivel y abierta del D. F. procrastina en la salud, 2015

\section{Causas}

No tengo tiempo

- . . . . . . . -

No tengo dinero

No hay quien me acompañe

Me da miedo saber qué es lo que tengo

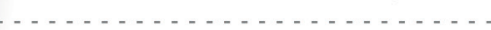

Mi pareja no me deja ir

No procrastinan
Esperan hasta el último momento

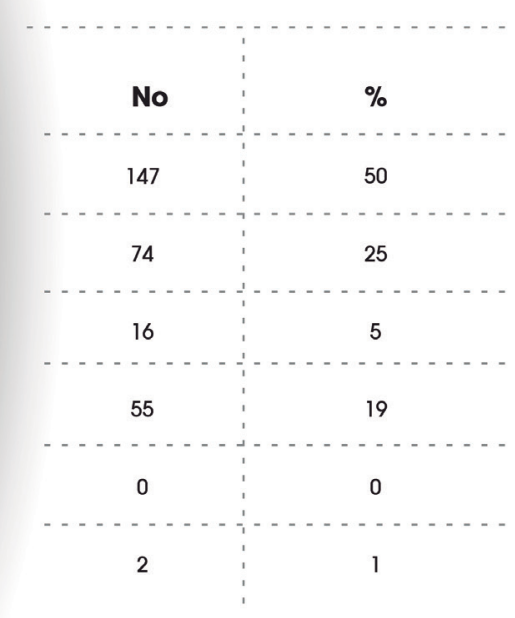

Aplazan su atención

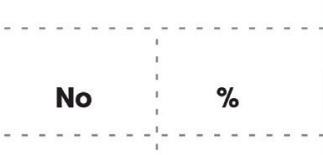

147

49

79
79

$11 \quad:$

11
59

0
0
2

164 IMN/Why Vol. 14. No. 3 septiembre - diciembre 2015 
El 25\% de la población tarda más de tres meses tan solo para decidir si se atiende o no, por lo que el 38\% solicita la atención de los servicios de salud cuando ya tiene una complicación.

Del 62\% de la población que dice atenderse antes de una complicación, solo el 55\% se realiza chequeo médico, de estos el 14\% se lo realizan cada tres años, lo que significa que es tiempo más que suficiente para desarrollar una enfermedad y/o complicación.

En lo que respecta al tratamiento, la población externó que tan solo el 15\% a veces sigue las indicaciones médicas, de estas el 75\% lo suspende porque ya se siente mejor, el 17\% no lo termina por cuestiones económicas. El 42\% de la población refirió que complementan su tratamiento con remedios caseros.

Tiempo que se tarda la población en decidir atenderse, 2015

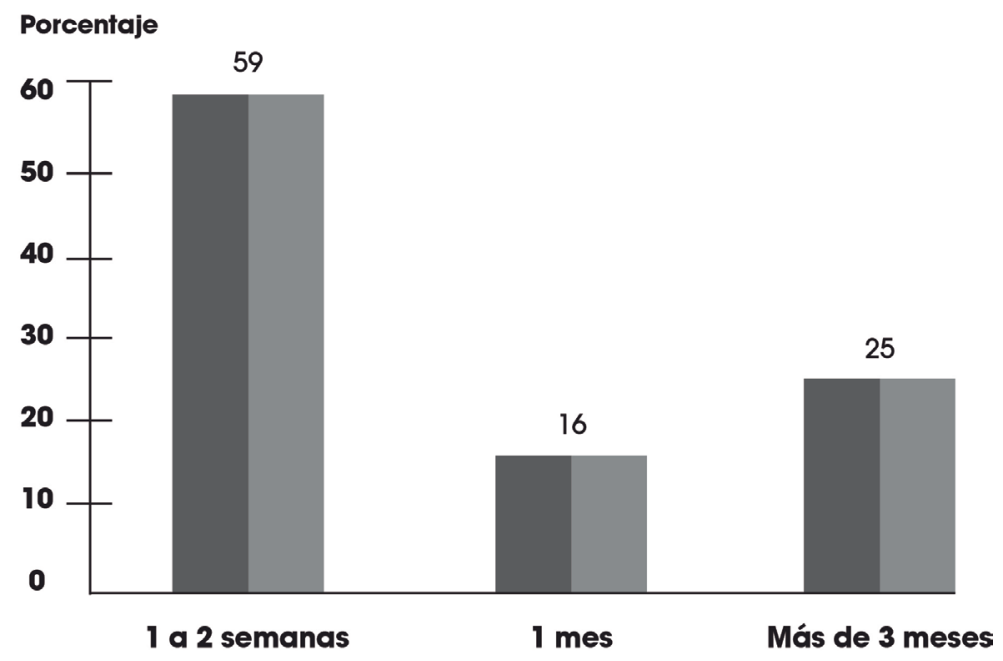

Al hacer el análisis bivariado se reportó una RP= 1.83, lo que significa que la probabilidad de procrastinar en salud es casi el doble de veces mayor en la población que tiene miedo a saber que enfermedad tiene con respecto a las que no tienen miedo o temor a saber su diagnóstico.

En cuanto a la medida de impacto el $45 \%$ de la población tiende a procrastinizar en su salud por miedo a saber lo que tiene, si nosotros les damos toda la información sobre su enfermedad podríamos disminuir la procrastinación en salud de la población.

En cuanto las personas que reportaron no tener dinero la RP fue de 1.39, la probabilidad de procrastinizar en su salud es 1.4 veces más cuando no tiene recursos económicos para atenderse.
Si aumentamos la seguridad social en la población podríamos disminuir la procrastinación en salud en un 28\% en la población mexicana.

Al analizar los datos de los servicios de salud, la población reporto no asistir a los servicios de salud por que no le resuelven su problema o enfermedad, al calcular la medida de efecto se encontró una $\mathrm{RP}=1.89$, por lo que la probabilidad de postergar su salud es casi dos veces mayor.

Si el personal de salud le da solución a las enfermedades entonces disminuiríamos el 47\% de procrastinación.

La probabilidad de procrastinizar en salud es de 1.5 más, porque pierden mucho tiempo esperando 
para ser atendidos, aun cuando existe un indicador de tiempo de espera este sigue siendo un problema en la calidad de atención.

Si se disminuye el tiempo de espera para la atención se podría evitar que el 33\% procrastine en su salud por el tiempo.

En ocasiones como los servicios de atención no le dan respuesta a su problema de salud acuden a los servicio privados los cuales en ocasiones son más caros, por lo que la población reporto que la probabilidad de procrastinar en su salud es de 1.4 veces más cuando se carece de recursos económicos a diferencia de los que no tienen este problema.

Si los servicios de salud fueran más eficientes y más accesiblemente económicos podríamos disminuir el 27\% de procrastinación.

Cabe señalar que al calcular la prueba de hipótesis Xi Mantel y Hansel, solo fue significativa cuando la población tiene miedo, quizá en las otras variables no se observa significancia estadística debido al tamaño de muestra.

\section{DISCUSIÓN}

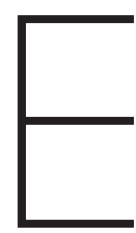

I seguro popular surge para brindar protección financiera a los mexicanos, ofreciendo una opción con criterios de aseguramiento público en materia de salud a familias y ciudadanos que por su condición laboral y socioeconómica no son derechohabientes de las instituciones de seguridad social. Cabe señalar que entre 2010 y 2012, incremento la afiliación al Seguro Popular por lo que aumentó el porcentaje así como el número de personas con acceso a los servicios de salud. Sin embargo la población reporto que procrastinan en su salud principalmente por la falta de dinero; el tiempo de espera para ser atendido, la carencia de medicamentos, entre otros. Esto implica que el crecimiento en la demanda de los servicios originados por una mayor afiliación ha ocasionado una reducción en la calidad de los servicios de salud con respecto a tiempos de espera y provisión de medicamentos.

A pesar de que la carencia por acceso a servicios de salud ha disminuido, los indicadores sobre el acceso oportuno a la atención médica y el acceso efectivo a los servicios de salud no han tenido una variación similar y en especial en los de servicios de salud preventivos existe un gran porcentaje de la población que no los utiliza. Como es la detección y control de enfermedades crónicas, factores de riesgo como el sobrepeso y obesidad.

Al comparar nuestros resultados con los reportados en los indicadores de calidad de la atención en 2010 por tipos de afiliación, podremos observar que los motivos de porque no se atienden son algunos de los que reportaron de porque procrastinan en su salud, los que más se destacan son la falta de dinero, el tiempo que hay que esperar para ser atendido, los altos costos de atención, tratamiento insuficiente y caros y al sentirse mejor no creen necesaria continuar con la atención (ver cuadro IV).

Cuadro 4. Comparación entre los datos resultantes de esta investigación y los indicadores sobre calidad en los servicios de salud, por tipo de afiliación, datos de la CONEVAL 2014.

\begin{tabular}{|c|c|c|c|c|c|c|}
\hline \multirow[t]{2}{*}{ Indicador } & \multicolumn{5}{|c|}{$\begin{array}{c}\text { Tipo de afiliación } \\
2010\end{array}$} & $\begin{array}{c}\text { Resultados de } \\
\text { esta investigación } \\
2015\end{array}$ \\
\hline & $\begin{array}{l}\text { Seguro } \\
\text { Popular }\end{array}$ & IMSS & ISSSTE & $\begin{array}{c}\text { No } \\
\text { afiliados }\end{array}$ & Otro & \\
\hline No tiene dinero & 35.18 & 12.61 & 22.74 & 3.32 & 56.92 & 26 \\
\hline Hay que esperar mucho para ser atendido & 20.80 & 7.43 & 14.31 & 9.16 & 47.90 & 27 \\
\hline
\end{tabular}

\section{6}

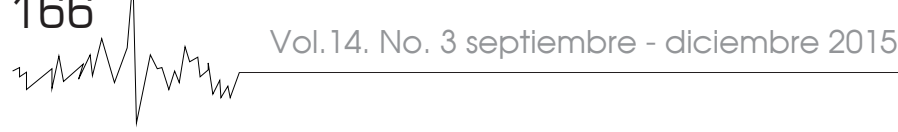




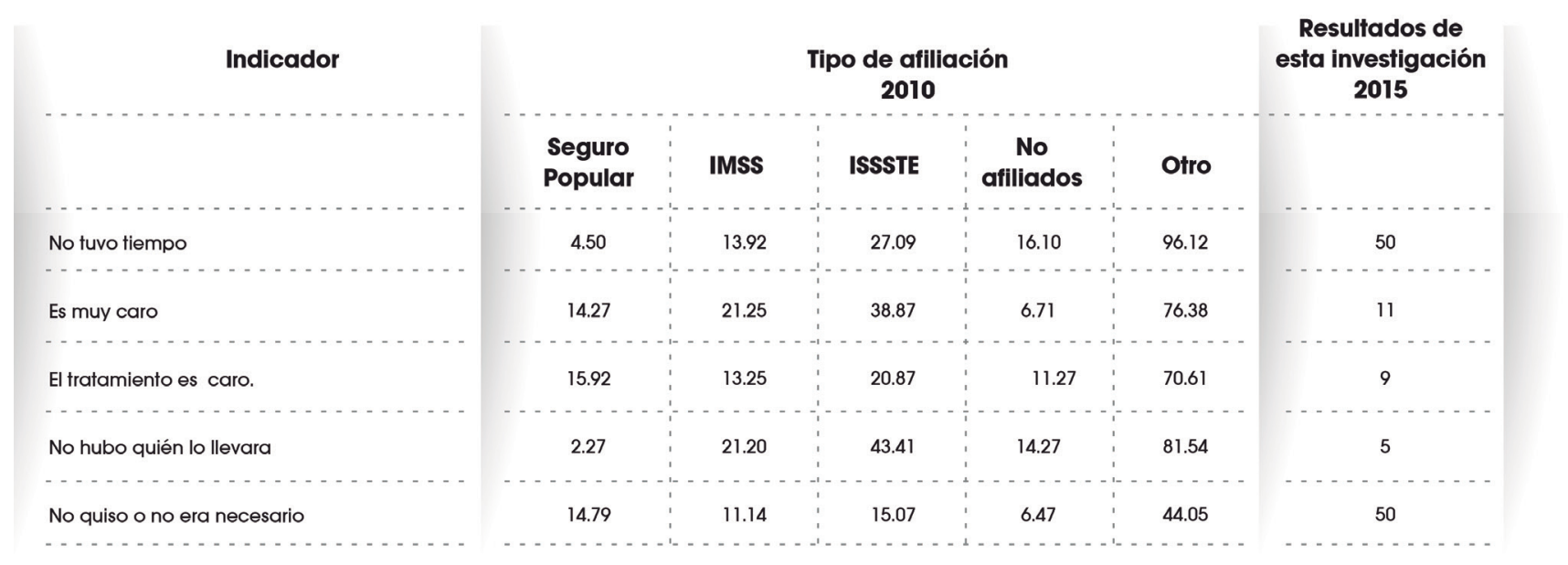

Fuente: Consejo Nacional de Evaluación de la Política de Desarrollo Social (CONEVAL). Indicadores de acceso y uso efectivo de los servicios de salud de afiliados al Seguro Popular. México DF. 2014; con datos del MCS-ENIGH 2008 y 2010 y la ENSANUT 2006 y 2012

Cabe señalar que algunos de nuestros resultados son muy similares al Seguro Popular quizás esto, se puede deber a la creciente afiliación y demanda de los servicios de salud, lo cual originando problemas en la atención de salud reflejándose en la calidad de atención. Así mismo en el 2015, se observa que persisten problemas y estos van en aumento más con respecto al Seguro Popular.

\section{CONCLUSIONES}

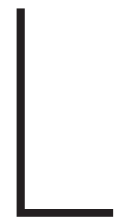

a procrastinación en salud se debe en gran medida a la falta de calidad que perciben los individuos de la atención de los servicios, los cuales están relacionados con: problemas económicos, tiempo de espera, resolución de la enfermedad y accesibilidad al tratamiento.

La calidad en la atención debe ser basada en actividades encaminadas a garantizar los servicios de salud accesibles y equitativos, con personal cualificado y con recursos disponibles, logrando la satisfacción del usuario con la atención recibida, para aumentar las expectativas que tiene el paciente con respecto a la atención y evitar seguir procrastinando en "salud" con el propósito de aumentar la esperanza y en particular la calidad de vida.

Por lo que es necesario que el personal de enfermería realice estrategias de fomento en la educación para la salud que transcienda a nivel teórico e informativo encaminadas a la toma de conciencia sobre la responsabilidad de desarrollar conductas saludables que tiendan a controlar o manejar los factores de riesgo modificables así como de su salud. El personal de enfermería toma un papel muy importante al ser este el primer contacto con la población quien informa y se comunica con ellos. Al explicar las enfermedades de una manera entendible y con alternativas para su mejoría dando confianza, con ello se disminuiría el miedo que tiene la población y se aumentaría la detección oportuna. En la promoción de la salud para los individuos y comunidades, se debe involucrar a estos para saber el proceso de salud enfermedad y que se tenga un mayor conocimiento para evitar procrastinar por no saber riesgos de la no atención. 
AGRADECIMIENTOS

Muchas gracias a las personas que de manera directa o indirecta me han ayudado en la realización de esta investigación. En especial a la M en C. Adela Alba Leonel por su asesoramiento, disciplina y apoyo en todo momento. Al Ingeniero Rubén Roa Ponce por la paciencia y comprensión, por los conocimientos que me han hecho crecer académica y personalmente.

\section{REFERENCIAS BIBLIOGRÁFICAS}

1. Angarita BLD, Aproximación a un concepto actualizado de la procrastonación. Rev. Iberoamericana de Psicología. 2012; 5(2): 85-94

2. Sánchez J, Procrastinación 101. 2012. (Consultado el 14/08/15). Disponible en: http://elgachupas.com

3. Steel P, The nature of procrastination: A meta-analytic and theoretical review of quintessential Self-Regulatory failure. Psychological Bulletin. 2007; 133 (1): 65-94

4. Contreras PH, Mori QE, Lam FN, Gil GE, Hinostroza CW, Rojas BD et al. Procrastinación en el estudio: exploración del fenómeno en adolescentes escolarizados. Rev. Perú, Epidemiolog. 2011 ; 15 (3): 1-5

5. Sobalvarro CC, La toma de decisión y la procrastinación. Universidad de San Carlos de Guatemala. XXXII Congreso Internacional de Psicología. Guatemala, 2009. 1-17.
6. Gaceta de la Escuela de Medicina Justo Sierra; Comprendiendo la procrastinación. México. 2009, 1-14.

7. Sánchez HAM, Procrastinación académica un problema en la vida universitaria. Studiositas. Edo. México. 2010; 5 (2): 87-94

8. Secretaria de Salud. Acuerdo por el que la Secretaría de Salud da a conocer las Reglas de operación e indicadores de gestión y evaluación del Programa Salud para Todos (Seguro Popular de Salud). México. (Consultado el 23/09/15). Disponible en: file:///D:/ SEGURO\%2OPOPULAR/SECRETARIA\%2ODE\%2OSAL UD\% 20..PROLONGACl\%F3N.html.

9. Consejo Nacional de Evaluación de la Política de Desarrollo Social (CONEVAL). Indicadores de acceso y uso efectivo de los servicios de salud de ailliados al Seguro Popular. México DF. 2014; 1-109 